\title{
Bir üniversite hastanesi parazitoloji laboratuvarında belirlenen intestinal ve hepatik parazitler
}

\author{
Intestinal and hepatic parasites determined in a university hospital parasitology laboratory
}

\author{
Zeynep Taş Cengiz'1, Yunus Emre Beyhan¹, Mutalip Çiçek², Hasan Yılmaz ${ }^{1}$
}

\section{ÖZET}

Amaç: Bu çalışmanın amacı Yüzüncü Yıl Üniversitesi Tıp Fakültesi Parazitoloji Laboratuarına başvuran hastalarda belirlenen bağırsak ve karaciğer parazitlerinin sıkığını ortaya koymaktır.

Yöntemler: Çalışma 2008 yılında yapılmış ve toplam 5985 dışkı örneği incelenmiştir. Dışkı örnekleri nativ-Lugol, sedimantasyon, flatasyon, trikrom boyama ve modifiye asit-fast boyama metotları ile değerlendirilmiştir. Entamoeba histolytica/E.dispar enfeksiyonu saptanan şüpheli hastaların dışkı örnekleri trikrom boyama metodu ile boyanmış, antijen yönünden ELISA ile de değerlendirilmiştir. ELISA metodu dışkı örnekleri Fasciola hepatica pozitif olan hastaların sonuçlarını doğrulamak için de kullanılmıştır.

Bulgular: Bu çalışmada 5985 kişinin \%29,6'sında intestinal parazitler belirlenmiştir. Çalışmada patojen parazitlerden Giardia intestinalis $(\% 9,4)$, bol Blastocystis hominis $(\% 5,5)$, Hymenolepis nana $(\% 1,7)$, Ascaris lumbricoides $(\% 1,2)$, Enterobius vermicularis $(\% 0,2$; dışkı bakısında), F.hepatica $(\% 0,1)$, Cyclospora cayetanensis $(\% 0,1)$, E. histolytica/E.dispar $(\% 0,06)$, Taenia saginata $(\% 0,05)$, Dicrocoelium dendriticum $(\% 0,05)$, Trichuris trichiura $(\% 0,03)$ ve Cryptosporidium spp. $(\% 0,02)$ belirlenmiştir.

Sonuç: Çalışmamızda yöremizde patojen intestinal parazitlerin hala düşük olamayan oranlarda görüldüğü ve parazitoz sorununun devam ettiği anlaşılmıştır.

Anahtar kelimeler: İntestinal ve hepatik parazitler, prevalans, Van, Türkiye

\begin{abstract}
Objective: The aim of this study is to present the prevalence of intestinal and hepatic parasites determined in Yüzüncü Yıl University Medical Faculty Parasitology Laboratory.
\end{abstract}

Methods: The study was performed in 2008, and a total of 5985 stool samples were examined. Stool samples were examined with native-Lugol, sedimentation, flotation, trichrome staining and modified acid-fast staining methods. The stool samples of patient suspected to have Entamoeba histolytica/E.dispar infection were stained by trichrome staining method and evaluated by ELISA method for the antigen. ELISA method was used to confirm the results of Fasciola hepatica positive patients in stool examination.

Results: In this study intestinal parasites were identified in $29.6 \%$ out of the 5985 people. In the study Giardia intestinalis (9.4\%), plenty Blastocystis hominis (5.5\%), Hymenolepis nana (1.7\%), Ascaris lumbricoides (1.2\%), Enterobius vermicularis ( $0.2 \%$; in the stool examination), F.hepatica $(0.1 \%)$, Cyclospora cayetanensis $(0.1 \%)$, E.histolytica/E.dispar $(0.06 \%)$, Taenia saginata $(0.05 \%)$, Dicrocoelium dendriticum (0.05\%), Trichuris trichiura (0.03\%) and Cryptosporidium spp. (0.02\%), pathogenic parasites, were detected.

Conclusion: In the study it is also understood that pathogenic intestinal parasites have still been reported at high rates and the problem of parasitosis continues in Van Province.

Key words: Intestinal and hepatic parasites, prevalence, Van, Turkey

${ }^{l}$ Yüzüncü Yıl Üniversitesi Tıp Fakültesi Parazitoloji Anabilim Dalı, Van, Türkiye
${ }^{2}$ Dicle Üniversitesi Tıp Fakültesi Mikrobiyoloji Anabilim Dalı, Diyarbakır, Türkiye

Yazışma Adresi /Correspondence: Zeynep Taş Cengiz, Yüzüncü Yıl Üniversitesi Tıp Fakültesi Parazitoloji Anabilim Dalı, 65080 Van, Türkiye Email: ztas72@hotmail.com Geliş Tarihi / Received: 25.06.2015, Kabul Tarihi / Accepted: 11.08.2015

Copyright @ Dicle Tıp Dergisi 2015, Her hakkı saklıdır / All rights reserved 


\section{GİRIŞ}

İntestinal parazitler özellikle sosyoekonomik seviyesi düşük olan ülkelerde su, besin, vektör ve kirli eşya aracılığıyla bulaşmakta ve enteritlere neden olmaktadır. Bu parazitozlara erişkinlere kıyasla çocuklarda daha sık rastlanmakta ve çocuklarda malnutrisyon oluşturarak bedensel ve zihinsel gelişime bozukluklarına da yol açabilmektedirler. İnsanlarda hepatik parazitler ise genellikle nadiren görülür ve bu parazitlerin sıklığg insanların beslenme alışkanlıkları, hijyen kuralları, parazitlerin dış ortamda biyolojisini devam ettirmesi için gerekli koşulların varlığı ile yakından ilişkilidir [1-4].

İntestinal parazitler ülkemizde Doğu Anadolu Bölgesi gibi sosyo-ekonomik düzeyi düşük olan bölgelerde daha sık rastlanır. Bölgemizde genel sosyo ekonomik durumun ve eğitim düzeyinin düşük olması, halkın paraziter hastalıklar konusunda yeterli bilgi ve bilince sahip olmamas1, hijyen kurallarına uyulmaması, içme suyunun genellikle açık su kanallarından temin edilmesi, su ve kanalizasyon alt yapısının yetersiz olması ve bazı su bitkilerinin çiğ olarak tüketilmesi bu yaygınlığın en önemli sebepleri arasındadır [1,3-5].

Bu çalışmanın amacı Yüzüncü Yı1 Üniversitesi Tıp Fakültesi Parazitoloji Laboratuarına başvuran hastalarda belirlenen bağırsak ve karaciğer parazitlerinin sıklığını ortaya koymaktır.

\section{YÖNTEMLER}

Bu çalışma 2008 yılında Yüzüncü Yı1 Üniversitesi Tıp Fakültesi Parazitoloji Laboratuarına başvuran 2785'i kadın, 3200'ü erkek olmak üzere toplam 5985 hasta üzerinde yürütüldü. $\mathrm{Bu}$ hastalardan 3565'ini 13 yaş ve daha küçük, 2420'sini 14 ve üzeri yaş grubu oluşturdu.

Dışk1 örneklerine nativ-Lugol, flatasyon (doymuş çinko sülfat solüsyonunda), sedimantasyon (formol-etil asetat solüsyonunda), modifiye asitfast (şüpheli örneklerde) ve trikrom boyama (şüpheli örneklerde) metodu uyguland1. Entamoeba histolytica/E.dispar şüpheli hastaların dışk1 örnekleri trikrom boyama metodu ile boyand 1 ve antijen yönünden ELISA (r-biopharm; RIDASCREEN $®$ Entamoeba (C 1701)) yöntemi ile de değerlendirildi. Dışk1 örneklerinde Fasciola hepatica ya da Dicrocoelium dendriticum yumurtası teşhis edilen hastalardan, dışkı incelemesinden bir hafta öncesine kadar karaciğer içeren bir yemek yiyip yemedikleri öğrenildi. Bu hastalar yalancı parazitlik ihtimaline karşı karaciğer içeren yemekler yememeleri gerektiği konusunda uyarıldıktan sonra, dışkıları üç gün üst üste tekrar incelendi. Ayrıca bu aşamadan sonra F.hepatica yönünden pozitif bulunan hastalardan kan alınarak ELISA (F.hepatica IgG ELISA; DRG International, Inc., USA) metodu ile de sonuçları değerlendirildi.

\section{İstatistiksel Analiz}

Parazit görülme oranları ile yaş grubu ve cinsiyet karşılaştırılmasında $Z$ testi ile oran karşılaştırması ve Fisher'in kesin olasılık testi (MINITAB Statistical Software; ver: 14) kullanıldı.

\section{BULGULAR}

Çalışmamızda intestinal parazitler 5985 hastanın \%29,6'sında belirlendi. Belirlenen 19 parazit türünün 12'si protozoon, 7'si helmintdi. Pozitif bulunan hastaların \%24,4'ünde bir, \%4,4'ünde iki, \%0,7'sinde üç, \%0,1'inde dört parazit türü belirlendi. 2785 k1zın \%27,1'inde, 3200 erkeğin \%31,9'unda bir ya da birden fazla parazit türü saptandı. 13 yaşından küçük 3565 hastanın \%32,7'sinde, 13 yaşından büyük 2420 hastanın \%25,1'inde parazit belirlendi. Teşhis edilen parazitlerin oranları sırasıyla şöyledir: Blastocystis hominis (\%16,6; her mikroskop alanındaki parazit sayıs1 5 ya da $>5$ (bol), \%5,5), Giardia intestinalis $(\% 9,4)$, Entamoeba coli $(\% 4,3)$, Hymenolepis nana $(\% 1,7)$, Ascaris lumbricoides $(\% 1,2)$, Chilomastix mesnili $(\% 0,8)$, Iodamoeba butschlii $(\% 0,5)$, Endolimax nana $(\% 0,3)$, Enteromonas hominis $(\% 0,2)$, Enterobius vermicularis $(\% 0,2$; d1şk1 bakısında), Trichomonas hominis $(\% 0,1)$, F.hepatica (\%0,1; sekiz hasta), Cyclospora cayetanensis $(\% 0,1)$, E.histolytica/E.dispar $(\% 0,06)$, Taenia saginata $(\% 0,05)$, D.dendriticum $(\% 0,05$; üç hasta), Trichuris trichiura $(\% 0,03)$, E.hartmanni $(\% 0,02)$, Cryptosporidium spp. (\%0,02; bir hasta) (Tablo 1).

Parazit görülme sıklığ ile hem yaş grupları hem de cinsiyetler arasındaki fark istatistiksel olarak anlamlı ( $\mathrm{p}=0.001$ ) bulundu. G.intestinalis ve A.lumbricoides görülme sıklığ 1 ile hem yaş $(\mathrm{p}=0.001)$ hem de cinsiyet (sirasiyla $p=0.001$ ve $p=0.008$ ) arasindaki fark, H.nana görülme sıklığı ile yaş $(\mathrm{p}=0.001)$ arasındaki fark istatistiksel olarak anlamlı bulundu (Tablo 1). 
Tablo 1. Yaş grubu ve cinsiyete göre parazit türlerinin prevalansı ve istatistik sonuçlar

\begin{tabular}{|c|c|c|c|c|c|c|c|c|c|c|c|c|}
\hline \multirow[t]{2}{*}{ Parazit türleri } & \multicolumn{2}{|c|}{$\begin{array}{c}\text { Toplam } \\
(n=5985)\end{array}$} & \multicolumn{2}{|c|}{$\begin{array}{c}\leq 13 \\
(n=3565)\end{array}$} & \multicolumn{2}{|c|}{$\begin{array}{c}\geq 14 \\
(n=2420)\end{array}$} & \multirow[t]{2}{*}{ p } & \multicolumn{2}{|c|}{$\begin{array}{c}\text { Kadın } \\
(n=2785)\end{array}$} & \multicolumn{2}{|c|}{$\begin{array}{c}\text { Erkek } \\
(\mathrm{n}=3200)\end{array}$} & \multirow[t]{2}{*}{ p } \\
\hline & $\mathrm{n}$ & $\%$ & $\mathrm{n}$ & $\%$ & $\mathrm{n}$ & $\%$ & & $\mathbf{n}$ & $\%$ & $\mathrm{n}$ & $\%$ & \\
\hline C.cayetanensis & 7 & 0,1 & 0 & 0 & 7 & 0,3 & $0.002^{*}$ & 3 & 0,1 & 4 & 0,1 & 0.845 \\
\hline G.intestinalis & 560 & 9,4 & 476 & 13,4 & 84 & 3,5 & $0.001^{*}$ & 215 & 7,7 & 345 & 10,8 & $0.001^{*}$ \\
\hline A.lumbricoides & 73 & 1,2 & 56 & 1,6 & 17 & 0,7 & $0.001^{*}$ & 23 & 0,8 & 50 & 1,6 & $0.008^{*}$ \\
\hline H.nana & 103 & 1,7 & 96 & 2,7 & 7 & 0,3 & $0.001^{*}$ & 41 & 1,5 & 62 & 1,9 & 0.163 \\
\hline E.histolytica/E.dispar & 4 & 0,06 & 3 & 0,08 & 1 & 0,04 & 0.652 & 2 & 0,07 & 2 & 0,06 & 0.890 \\
\hline B.hominis & 993 & 16,6 & 569 & 16 & 424 & 17,5 & 0.114 & 437 & 15,7 & 556 & 17,4 & 0.08 \\
\hline T.trichiura & 2 & 0,03 & 1 & 0,03 & 1 & 0,04 & 0.79 & 2 & 0,07 & 0 & 0 & 0.216 \\
\hline$T$ saginata & 3 & 0,05 & 0 & 0 & 3 & 0,1 & 0.066 & 0 & 0 & 3 & 0,09 & 0.253 \\
\hline F.hepatica & 8 & 0,1 & 3 & 0,1 & 5 & 0,2 & 0.282 & 6 & 0,2 & 2 & 0,1 & 0.157 \\
\hline Parazitli hastalar & 1774 & 29,6 & 1166 & 32,7 & 608 & 25,1 & $0.001^{*}$ & 754 & 27,1 & 1020 & 31,9 & $0.001^{*}$ \\
\hline
\end{tabular}

*istatistiksel olarak anlamlı

\section{TARTIŞMA}

Ülkemizde iklim ve çevre koşullarının uygun olmas1, altyapı eksikliği, bazı yörelerde düşük sosyoekonomik durum, halkın paraziter hastalıklar konusunda yeterince bilgi ve bilince sahip olmaması ve özellikle çocukların hijyen kurallarına uymaması gibi nedenlerle bağırsak parazitlerine sıklıkla rastlanmaktadır [1,2].

Ülkemizde intestinal parazitlerin prevalansını belirlemek için genellikle hastanelere başvuran hasta verilerinden faydalanılmıştır. Farklı hastanelerde yürütülen çalışmalarda yörelere göre değişen yay1lış oranları belirlenmiştir.

Ege Üniversitesi Tip Fakültesi Parazitoloji Laboratuarında 2005 yılı boyunca 3925 hastanın gaita ve selofan bant preparatları incelenmiş dışkı örneklerinin \%15,03'ünde bağırsak parazitlerine rastlanmıştır. Çalışmada en sık belirlenen beş tür B.hominis $(\% 4,96)$, Cyclospora spp. $(\% 1,91)$, E.vermicularis $(\% 1,86)$ ve G.intestinalis $(\% 1,78)$ olmuştur [6].

Uludağ Üniversitesi Tıp Fakültesi Parazitoloji Laboratuarında yürütülen bir çalışmada 32,346 dışk1 örneği incelenmiştir. İncelenen örneklerin $\% 8,14$ 'ü parazit yönünden pozitif bulunmuş ve pozitif olguların \%46,32'sini kadın, \%53,68'ini erkekler oluşturmuştur. Çalışmada G.intestinalis \%3,63, E.vermicularis \%3,41 (10,897 selofan bant örneğinden), E.histolytica/E.dispar \%0,35, A.lumbricoides $\% 0,22$, Taenia spp. $\% 0,089$, D.dentriticum $\% 0,021$,
T.trichiura \%0,018, H.nana \%0,012, S.stercoralis $\% 0,006$ oranında saptanmıştır [7].

Kocaeli Üniversitesi Tıp Fakültesi Parazitoloji Laboratuarına başvuran 5178 kişiden dışkı örneği alınmıştır. İncelenen dışkı örneklerinin \%10,67'sinde bir veya birden fazla bağırsak paraziti saptanmış, pozitif olguların \%39,8'inin kadın, \%60,2'sinin ise erkek olduğu görülmüştür. Çalışmada G.intestinalis $\% 24,95$, E.histolytica/E.dispar \%3,43, B.hominis $\% 20,97$, E.vermicularis \%23,32 (4560 selofan bant örneğinden) ve Taenia spp. \%3,07 oranında belirlenmiştir [8].

Eskişehir Osmangazi Üniversitesi Tıp Fakültesi Parazitoloji Laboratuarına başvuran 34,733 hastaya ait dışk1 örneğinin \%3,6'sında bir veya daha fazla türde parazit varlığ tespit edilmiştir. Çalışmada parazit saptanan olguların \%52,5'inin kadın, $\% 47,5$ 'inin erkek; en s1k rastlanan ilk üç türün $E$. histolytica/E.dispar (\%31), G.intestinalis (\%19) ve B.hominis (\%7) olduğu bildirilmiştir. Ayrıca incelenen 1252 selofan lam preparatının $\% 2,3$ 'ünde $E$. vermicularis, $\% 0,8$ 'inde T.saginata, $\% 0,4$ 'ünde ise S.stercoralis saptandığı belirtilmiştir [9].

Mustafa Kemal Üniversitesi Tıp Fakültesi Parazitoloji Laboratuarına başvuran 3679 kişinin dışkı örneği incelenmiș, erkeklerin \%34,23'ü, kadınların $\% 65,76$ 's1, tüm hastaların ise \%21,03'ü intestinal parazitler yönünden pozitif bulunmuştur. İncelenen dışk1 örneklerinde G.intestinalis \%31,5, B.hominis $\% 25,3$, E.histolytica /E.dispar \%13,4, E.vermicula- 
ris $\% 8,72$ (1720 selofan bant örneğinden), D.dentriticum \%1,2, A.lumbricoides \%1, H.nana \%1,4, S.stercoralis $\% 0,2$ ve T.saginata $\% 0,7$ oranında saptanmıştır [10].

Erciyes Üniversitesi Tıp Fakültesi Parazitoloji Laboratuarında toplam 28,911 dişkı örneğinin $\% 24,13$ 'ünde (\%52,9'u kadın, \%47,1'i erkek) intestinal parazitlere rastlanmıştır. Çalışmada B.hominis'e \%19,72, G.intestinalis'e \%1,96, E.histolytica/E.dispar'a \%0,87, E.vermicularis'e \%0,2 (7.164 selofan bant örneğinden), Taenia spp.'ye \%0,12, A.lumbricoides'e \%0,12 ve H.nana'ya \%0,06 oranında rastlanmıştır [11].

Firat Üniversitesi Fırat Tıp Merkezi Parazitoloji Laboratuarında yapılan bir çalışmada 1218 hastaya ait dışk1 örneğinin \%17,24'ünde parazit saptanmıştır. Bu çalışmada B.hominis' in \%26,66, G.intestinalis' in \%24,76, E.vermicularis' in \%8,57, E.histolytica/E.dispar'1n \%3,33, T.saginata'nın $\% 2,38$ ve H.nana'nın \%0,47 oranında yaygın olduğu görülmüştür [12].

Cumhuriyet Üniversitesi Tip Fakültesi Parazitoloji Laboratuarına başvuran toplam 5057 kişinin dışkı örneği incelenmiş, bağırsak parazitleri kadınların \%4,5'inde, erkeklerin \%5,9'unda olmak üzere tüm hastaların \%10,5'inde saptanmıştır. Çalışmada E.vermicularis \%5,4 (1313 selofan bant örneğinden), G.intestinalis \%3,7, E.histolytica/dispar $\% 2,4$, T.saginata \%0,6, B.hominis \%0,4, A.lumbricoides $\% 0,05, H$.nana $\% 0,01$ ve T.trichura $\% 0,01$ oranında tespit edilmiştir [13].

Yüzüncü Yıl Üniversitesi Tıp Fakültesi Parazitoloji Laboratuarına başvuran 0-13 yaş grubu çocuklara ait 3505 dışk1 örneğinin \%22,22'sinde bir veya daha fazla sayıda bağırsak paraziti saptanmış olup çalışmada E.histolytica'nın \%7,96, A.lumbricoides'in \% $\%, 81$, G.intestinalis' in \%4,93, E.vermicularis'in \%0,28, H.nana'nın \%0,11, $H$. diminuta'nın \%0,05, T.saginata'nın \%0,08, T.trichiura'nın \%0,11 ve B.hominis' in \%0,05 oranında yaygın olduğu bildirilmiştir [3]. Aynı laboratuarda 14 ve üzeri yaş grubu hastalara ait 3534 dışkı örneğinin 922'sinde bir veya birden fazla parazit türü tespit edilmiş, bunların 462'sinin kadın, 460'ının erkek olduğu bildirilmiştir. Çalışmada A.lumbricoides \%12,99, E.histolytica \%11,18, G.intestinalis $\% 1,75$, E.vermicularis $\% 0,48$, T.saginata $\% 0,34$,
H.nana $\% 0,20$, T.trichiura $\% 0,14$, H.diminuta ve S.stercoralis $\% 0,09$, çengelli solucan ve F.hepatica $\% 0,03$ oranında belirlenmiştir [4].

Yüzüncü Y11 Üniversitesi Araştırma ve Uygulama Hastanesi Parazitoloji Laboratuarında 3037'si kadın 3230'u erkek olmak üzere toplam 6267 kişiye ait dışkı örneği incelenmiş, kadınların \%28'inde, erkeklerin \%29'unda, tüm hastaların ise \%28,5'inde bir ya da birden fazla parazit türüne rastlamışlardır. Çalışmada B.hominis \%15,4, G.intestinalis \%6,6, B.hominis \%3,2 (bol), H.nana \%1,3, A.lumbricoides \%0,5, E.histolytica/E.dispar \%0,1, C.cayetanensis \%0,1, E.vermicularis \%0,1, D.dendriticum $\% 0,03$, T.saginata $\% 0,03$ ve T.trichiura $\% 0,02$ oranında saptanmıştır [5].

Yaptığımız bu çalışmada ise intestinal parazitler hastaların \%29,6'sında belirlenmiştir. Bu parazitler erkeklerde kadınlara göre, 13 yaşından küçük olanlarda 13 yaşından büyük olanlara göre daha yüksek oranda görülmüştür ayrıca parazit görülme sıklığı ile hem yaş grupları hem de cinsiyetler arasındaki fark istatistiksel olarak anlamlı bulunmuştur. Çalışmamızda en sık belirlenen üç patojen parazit türü G.intestinalis $(\% 9,4)$, bol B.hominis $(\% 5)$ ve H.nana $(\% 1,7)$ olmuş, insanlarda nadir görülen hepatik parazitlerden F.hepatica sekiz, D.dendriticum ise üç hastada saptanmıştır.

Sonuç olarak, çalışmamızda yöremizde patojen intestinal parazitlerin hala düşük olmayan oranlarda görüldüğü ve parazitoz sorununun devam ettiği anlaşı1mıştır.

\section{KAYNAKLAR}

1. Özcel MA, Özbel Y, Ak M. Özcel'in T1bbi Parazit Hastalıkları. Meta Basım, İzmir; 2007.

2. Sayg1 G. Temel T1bbi Parazitoloji. 1. Bask1, Esnaf Ofset Matbaac1lık, Sivas; 1998.

3. Yilmaz H, Cesur Y, Ozkaya E, ve ark. Distribution of intestinal parasites 0-13 years old children admitted to Parasitology Laboratory of School of Medicine, Yüzüncü Y1l University. Turkiye Parasitol Derg 1997;21:387-390.

4. Yilmaz H, Turkdogan K, Berktas M, et al. Distribution of intestinal parasites among 14 years old and older patients admitted to Parasitology Laboratory of Medicine Faculty. Turkiye Parasitol Derg 1997;21:49-54.

5. Yılmaz H, Taş-Cengiz Z, Ceylan A, ve ark. Yüzüncü Y1l Üniversitesi Araştırma ve Uygulama Hastanesi Parazitoloji Laboratuarına 2009 yılında başvuran kişilerde bağırsak parazitlerinin dağılımı. Türkiye Parazitol Derg 2012;36:105108 . 
6. Değirmenci A, Sevil N, Güneș K, ve ark. Ege Üniversitesi Tıp Fakültesi Hastanesi Parazitoloji Laboratuvarında 2005 yılı boyunca saptanan bağırsak parazitlerinin dağılımı. Türkiye Parazitol Derg 2007;31:133-135.

7. Alver O, Özakın C, Yılmaz E, ve ark. Uludağ Üniversitesi Tıp Fakültesinde farklı yıllarda bağırsak parazit dağılımlarının belirlenmesi. Türkiye Parazitol Derg 2005;29:193199.

8. Sönmez Tamer G, Çalışkan Ş, Willke A. Kocaeli Üniversitesi Tıp Fakültesi Parazitoloji Laboratuvarına başvuran hastalarda bağırsak parazitlerinin dağılımı. Türkiye Parazitol Derg 2008;32:126-129.

9. Doğan N, Demirüstü C, Aybey A. Eskişehir Osmangazi Üniversitesinin beş yıllık bağırsak paraziti prevalansının türlere ve cinsiyete göre dağılımı. Türkiye Parazitol Derg 2008;32:120-125.
10. Culha G. Mustafa Kemal Üniversitesi Tıp Fakültesi Parazitoloji Laboratuvarına başvuran hastalarda bağırsak parazitlerinin dağ 11 ımı. Türkiye Parazitol Derg 2006;30:302-304.

11. Yaman O, Yazar S, Özcan H, et al. 2005-2008 yılları arasında Erciyes Üniversitesi Tıp Fakültesi Parazitoloji Laboratuvarına başvuran hastalarda bağırsak parazitlerinin dağılımı. Türkiye Parazitol Derg 2008;32:266-270.

12. Kuk S, Erensoy A, Keleştemur N. Son bir yıl içinde Firat Üniversitesi Fırat Tıp Merkezi Parazitoloji Laboratuvarında koproparazitolojik inceleme sonuçları. Fırat Tıp Derg 2006:11:113-115.

13. Değerli S, Özçelik S, Çeliksöz A. Cumhuriyet Üniversitesi Tıp Fakültesi Parazitoloji Laboratuvarına başvuran hastalarda bağırsak parazitlerinin dağılımı. Türkiye Parazitol Derg 2005;29:116-119. 